\section{Periocular implantation cysts: a late complication of ophthalmic surgery}

\author{
Abstract \\ Purpose To describe the clinical \\ characteristics for a group of patients \\ presenting with acquired implantation cysts \\ following periocular surgery. \\ Methods Retrospective, non-comparative \\ series of patients who underwent surgery at \\ Moorfields Eye Hospital for orbital \\ implantation cysts, with review of clinical \\ case notes and imaging. \\ Results Twenty-three patients (13 male; \\ 57\%) were treated between 1997 and 2011 \\ with cystic lesions that presented at an \\ average interval of 22.7 years after previous \\ periocular surgery. The age at presentation \\ ranged from 14 to 76 years (mean 45.3; \\ median 46 years). Five patients had a history \\ of prior enucleation or evisceration (with ball \\ implantation in $4 / 5$ cases) and six had \\ undergone orbital fracture repair with either \\ a silicone or titanium plate. Eight patients \\ had prior strabismus surgery, three had \\ retinal detachment repair with either a scleral \\ buckle or vitrectomy, and one patient had \\ repair of a conjunctival laceration in \\ childhood. All but one patient underwent \\ either wide marsupialisation or complete \\ excision of the implantation cysts, with no \\ record of recurrence. Visual acuity improved \\ after surgery in a half of the patients, \\ whereas remaining unchanged in the \\ remainder. \\ Conclusion Orbital implantation cysts \\ constitute a relatively rare complication of \\ periocular surgery and can present decades \\ after the causative procedure. \\ Eye (2014) 28, 1004-1007; doi:10.1038/eye.2014.111; \\ published online 13 June 2014
}

\section{Introduction}

Orbital implantation cysts rarely occur after periocular surgery. They typically result from
GK Kalantzis, DH Verity and GE Rose

the misplacement of conjunctival epithelium into orbit fat ${ }^{1}$ and can occur after eye removal (with or without ball implantation), strabismus surgery, or retinal detachment repair with scleral buckling. Problematic cystic spaces can also occur around implant materials, although these cysts will often not have an epithelial lining.

We present a large series of orbital implantation cysts of diverse periocular and orbital procedures, but similar aetiology, and describe their clinical characteristics and management.

Materials and methods

A retrospective review of the clinical case notes and, where available, imaging was performed for patients at Moorfields Eye Hospital operated between 1997 and 2011 for orbital implantation cysts, and a review of the English ophthalmic literature performed. All orbital pathology is data-based within the Service.

This study followed the principles of the Declaration of Helsinki and the local IRB/Ethics Committee ruled that approval was not required for this study. Informed consent was provided by all patients undergoing surgery.

Results

\section{Clinical case-reports}

Case 1 A 50-year-old man, who had undergone repair of a left orbital floor fracture with placement of an implant 25 years previously, presented with slowly progressive proptosis ( $3 \mathrm{~mm})$, hyperglobus $(2 \mathrm{~mm})$, and restricted ocular movements with diplopia. Orbital CT identified a soft-tissue mass on a sclerotic orbital floor. At surgery, a large implant-associated cyst - filled with mucoid secretions - was found surrounding a silicone sheet implant and both the implant and surrounding cyst were excised. 
Histology revealed a fibrous cyst with mucus-secreting epithelium-possibly of conjunctival or respiratory origin-with intramural haemosiderin and cholesterol granulomas consistent with prior haemorrhage.

Case 2 Twenty-six years after right enucleation for a ruptured globe, with secondary acrylic ball implantation a few weeks later, a 46-year-old man presented with vague ache within his socket, increasing lower lid swelling and some instability of his prosthesis. CT showed a round, moderately-radiolucent lesion lying supero-temporal to the implant. The inclusion cyst was widely marsupialised to the surface, and histology was shown to be of conjunctival origin, with underlying fibrosis and a few smooth muscle fibres.

Case 3 A 49-year-old man presented with many years of increasing displacement of his left globe. He had sustained a major fracture of the orbital roof 23 years earlier, which had been repaired with placement of an implant. His left eye was markedly displaced (12 mm relative proptosis and $5 \mathrm{~mm}$ hypoglobus) with imaging revealing a large implantation cyst that was excised together with an enclosed silicone sheet (Figure 1). Histology revealed a fibrous cyst containing a ciliated pseudo-columnar respiratory epithelium.

Case 4 A 28-year-old lady presented with intermittent ocular irritation 25 years after lateral rectus recession due to a gradually enlarging left conjunctival cystic lesion; the lesion was also associated with mild restriction in abduction (Figure 2). She underwent uncomplicated excision of a conjunctival inclusion cyst tightly bound to the lateral rectus insertion.

\section{Group characteristics}

Twenty-three patients (13 male; 57\%) presented at a median age of 46 years (mean 45.3; range 14-76 years), with the interval between previous surgery and presentation ranging from 1 to 50 years (median 25 years). Five had undergone ocular removal (with ball implantation in 4), six had orbital fracture repair with a silicone or titanium implant, eight had a history of prior strabismus surgery, three had retinal detachment repair (either a scleral buckle or vitrectomy) and a single patient had required repair of a conjunctival laceration during childhood. All except one patient underwent either marsupialisation or complete excision of the implantation cyst, with no record of recurrence at up to 56 months follow-up (mean 14, median 24, range 3-56 months). All cysts had a fibrotic wall of variable thickness, with most showing mucin-secreting conjunctival epithelium or, more rarely, pseudostratified

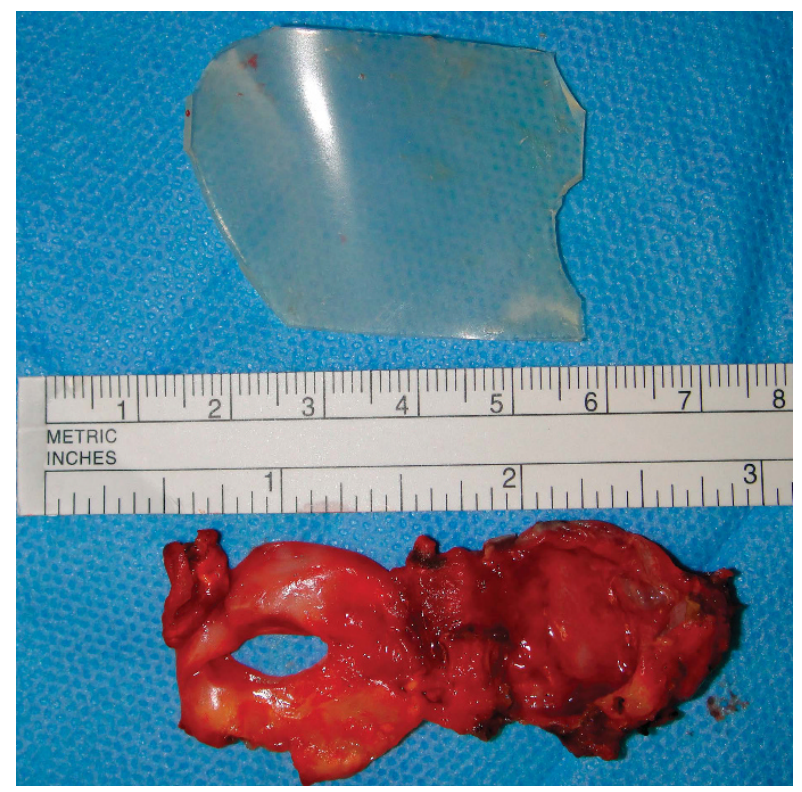

Figure 1 Excised fibrous cyst and silicone sheet removed from an area of orbital roof repair performed 23 years before (Patient 11; Table 1).

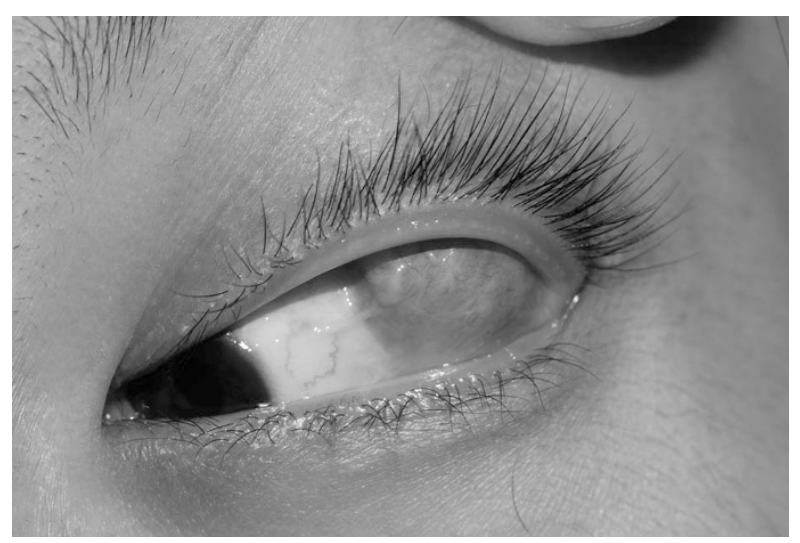

Figure 2 Patient presenting with a large orbital conjunctival inclusion cyst overlying the lateral rectus insertion at 25 years after strabismus surgery (Patient 12; Table 1).

ciliated respiratory epithelium (Table 1 ). The visual acuity changed in a half $(11 / 22)$ of the patients undergoing surgery, this always being an improvement (Figure 3).

Only one patient, presenting with a cyst and a small exotropia after multiple strabismus operations 29 years earlier, declined surgery.

\section{Discussion}

Orbital implantation cysts are usually lined by conjunctival $^{2}$ or, more rarely, pseudostratified columnar ciliated respiratory epithelium ${ }^{3}$ The latter, being of 
sinus origin, is probably drawn into the orbit during retrieval of prolapsed orbital contents at the time of fracture repair, whereas conjunctival implantations are probably due to direct displacement from the ocular surface. ${ }^{4-6}$ Most cysts around silicone implants are fibrous, with late occurrence of intracystic haemorrhage, although true epithelium-lined cysts do rarely occur. ${ }^{3,7,8}$ In a 10 -year study of the

Table 1 Change of visual acuity in the affected eye of 22 patients undergoing removal of periocular implantation cysts

\begin{tabular}{lcc}
\hline Patient number & $\begin{array}{c}\text { Vision prior } \\
\text { to surgery }\end{array}$ & $\begin{array}{c}\text { Vision at latest } \\
\text { follow-up }\end{array}$ \\
\hline 1 & $6 / 9$ & $6 / 9$ \\
2 & $6 / 12$ & $6 / 9$ \\
3 & $6 / 12$ & $6 / 6$ \\
4 & $6 / 6$ & $6 / 6$ \\
5 & $\mathrm{NPL}$ & $\mathrm{NPL}$ \\
6 & $6 / 18$ & $6 / 18$ \\
7 & $6 / 24$ & $6 / 18$ \\
8 & $\mathrm{NPL}$ & $\mathrm{NPL}$ \\
9 & $6 / 18$ & $6 / 9$ \\
10 & $6 / 6$ & $6 / 5$ \\
11 & $6 / 36$ & $6 / 12$ \\
12 & $6 / 5$ & $6 / 5$ \\
13 & $6 / 12$ & $6 / 9$ \\
14 & $\mathrm{NPL}$ & $\mathrm{NPL}$ \\
15 & $6 / 6$ & $6 / 6$ \\
16 & $\mathrm{NPL}$ & $\mathrm{NPL}$ \\
17 & $\mathrm{NPL}$ & $\mathrm{NPL}$ \\
18 & $6 / 7.5$ & $6 / 6$ \\
19 & $6 / 9$ & $6 / 9$ \\
20 & $6 / 12$ & $6 / 9$ \\
21 & $6 / 18$ & $6 / 9$ \\
22 & $6 / 12$ & $6 / 9$ \\
23 & & $6 / 24$ (not operated) \\
\hline
\end{tabular}

complications of alloplastic implants, only two patients were reported to have developed cysts, these related to partial absorption of Gelfilm implants. ${ }^{9}$ The other sporadic reports of surgical implantation cysts involve patients who had previous enucleation, ${ }^{10}$ retinal detachment surgery with scleral buckling, ${ }^{2}$ or strabismus surgery; ${ }^{11}$ in all cases, complete excision of the cysts was curative. Visual functions improved after surgery in a half of our series of patients, with no deterioration in the remainder-this improvement highlighting the value of such surgery in relieving distortion of the globe or possibly pressure on the optic nerve. $^{12}$

The current study of 23 patients emphasises the diverse causes of orbital implantation cysts. A thorough history of all prior periocular surgery-whether recent or several decades earlier-will alert the clinician to the probable aetiology of an apparently 'new' cyst. In this study, the maximum interval before presentation was 50 years, with the median interval being 25 years. The rarity of such cysts might suggest that implanted epithelial elements during periocular surgery typically do not remain viable. The exceptional delay in presentation described in this (and other) series would suggest that, where implanted epithelium does continue to proliferate, growth is typically slow and 'exponential' - the individual cases described showing that very large cysts can present with a short history as compared with the interval since original surgery. As no cases of implantation cyst were identified in the absence of surgery, it is likely that all occurred because of the prior surgical manipulation or trauma. All of our patients were cured by excision of the cyst or, where on the surface of a socket, by wide marsupialisation to the surface.

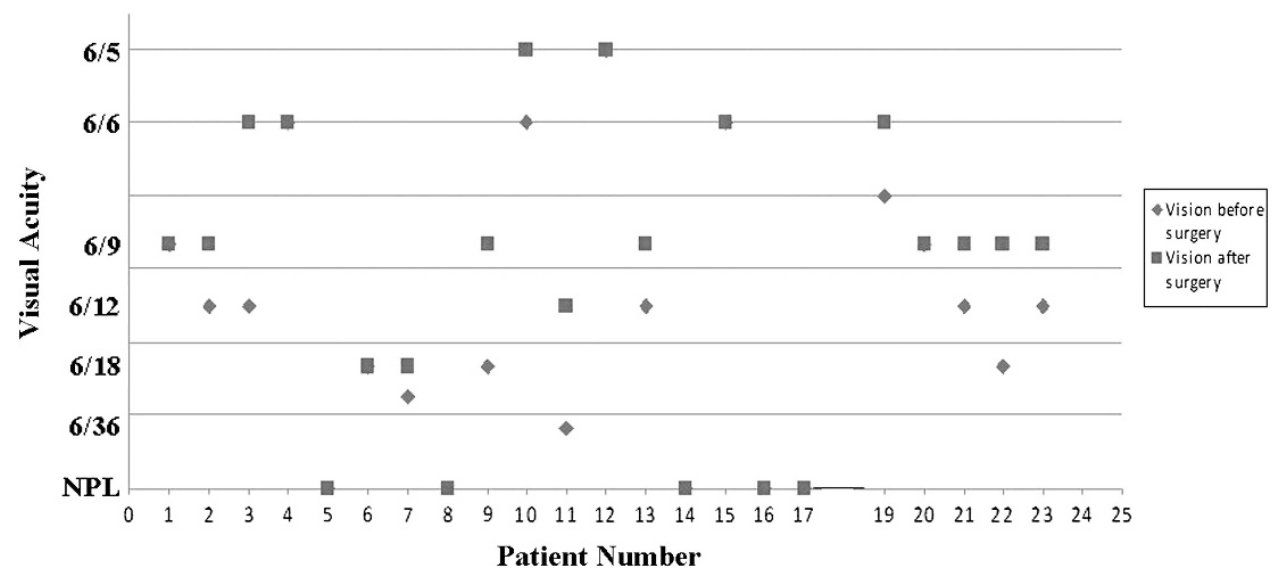

Figure 3 Preoperative and postoperative Snellen acuities in the operated eye of 22 patients undergoing excision of a periocular implantation cyst, patient 18 not undergoing surgery. 
Summary

\section{What was known before}

- Orbital implantation cysts rarely occur after periocular surgery.

- They typically result from misplacement of conjunctival epithelium into orbit fat.

What this study adds

- We present the largest series of orbital implantation cysts of diverse periocular and orbital procedures, but similar aetiology.

- Visual functions improved after surgery in half of the patients and remained stable for the rest. This improvement highlighting the value of such surgery in relieving distortion of the globe or possibly pressure on the optic nerve.

\section{Conflict of interest}

The authors declare no conflict of interest.

\section{Acknowledgements}

Professor Geoffrey Rose receives some funding from the National Institute of Health Research Biomedical Research Centre at Moorfields Eye Hospital NHS Foundation Trust and UCL Institute of Ophthalmology. The sponsor or funding organisation had no role in the design or conduct of this research.

\section{References}

1 Morax S, Herdan ML, Chouard B. Orbital cysts caused by conjunctival inclusion occurring after orbito-oculo-palpebral surgery. J Fr Ophtalmol 1987; 10: 41-49.
2 Johnson DW, Bartley GB, Garrity JA, Robertson DM. Massive epithelium-lined inclusion cysts after scleral buckling. Am J Ophthalmol 1992; 113: 439-442.

3 Neves RB, Yeatts RP, Martin TJ. Pneumo-orbital cyst after orbital fracture repair. Am J Ophthalmol 1998; 125: 879-880.

4 Tan CS, Ang LP, Choo CT, Cheah ES, Chee SP. Orbital cysts lined with both stratified squamous and columnar epithelia: a late complication of silicone implants. Ophthal Plast Reconstr Surg 2006; 22: 398-400.

5 Ma'luf RN. Cyst formation associated with porous polyethylene orbital floor implant. Orbit 2010; 29: 343-345.

6 Hillier RJ, Osborne SF, Leatherbarrow B. Epithelial inclusion cyst associated with a porous polyethylene orbital floor implant. Ophthal Plast Reconstr Surg 2009; 25: 238-239.

7 Schmidt BL, Lee C, Young DM, O'Brien J. Intraorbital squamous epithelial cyst: an unusual complication of Silastic implantation. J Craniofac Surg 1998; 9: 452-455.

8 Tahhan M, Alkhardaji F, Durrani OM, Price NJ. Intraorbital epithelial cyst formation: a rare complication of silastic implantation. Arch Ophthalmol 2002; 120: 1768-1769.

9 Jordan DR, Onge P, Anderson RL, Patrinely JR, Nerad JA. Complications associated with alloplastic implants used in orbital fracture repair. Ophthalmology 1992; 99: 1600-1608.

10 Jünemann A, Holbach LM. Epithelial giant inclusion cyst 50 years after enucleation without orbital implant. Klin Monbl Augenheilkd 1998; 212: 127-128.

11 Song JJ, Finger PT, Kurli M, Wisnicki HJ, Iacob CE. Giant secondary conjunctival inclusion cysts: a late complication of strabismus surgery. Ophthalmology 2006; 113: $1045-1049$.

12 Harris GJ, Beatty RL, Massaro BM, Lewandowski MF. Conjunctival implantation cyst of the orbit: Transient visual loss with pregnancy. Arch Ophthalmol 1989; 107: 924-927. 